\title{
Investigation of a Polynomial Matrix Generalised EVD for Multi-Channel Wiener Filtering
}

\author{
Jamie Corr ${ }^{1, \dagger}$ Jennifer Pestana ${ }^{2}$, Stephan Weiss ${ }^{1}$, Soydan Redif ${ }^{3}$ and Marc Moonen ${ }^{4}$ \\ ${ }^{1}$ Dept. Electronic \& Electrical Engineering, University of Strathclyde, Glasgow Scotland \\ ${ }^{2}$ Dept. Mathematics \& Statistics, University of Strathclyde, Glasgow Scotland \\ ${ }^{3}$ Electrical \& Electronic Engineering Dept. European University of Lefke, Cyprus \\ ${ }^{4}$ Dept. Electrical Engineering, KU Leuven, Leuven, Belgium
}

\begin{abstract}
State of the art narrowband noise cancellation techniques utilise the generalised eigenvalue decomposition (GEVD) for multichannel Wiener filtering which can be applied to independent frequency bins in order to achieve broadband processing. Here we investigate the extension of the GEVD to broadband, polynomial matrices, akin to strategies that have already been developed by McWhirter et. al on the polynomial matrix eigenvalue decomposition (PEVD).
\end{abstract}

\section{Extended Summary}

Motivation. Recently the scalar generalised eigenvalue decomposition (GEVD) has been utilised to enable low rank approximation in multichannel Wiener filters (MWF) $[3,8]$. At the stage where the signal only covariance matrix is estimated, the estimation process artificially increases the rank of the matrix. To avoid using matrices with higher rank than required, the covariance matrix gets replaced by a low rank approximation [3]. Methods using both the conventional eigenvalue decomposition (EVD) and GEVD have been explored in [8] with results showing the GEVD based approach performs better as it effectively selects the modes with the highest SNR.

For a broadband array vector $\mathbf{x}[n]$, where explicit lags $\tau$ need to be taken into accout, the space-time matrix is defined as $\mathbf{R}[\tau]=\mathcal{E}\left\{\mathbf{x}[n] \mathbf{x}^{\mathrm{H}}[n-\tau]\right\}$. Its $z$-transform $\boldsymbol{R}(z) \bullet \cdots \mathbf{R}[\tau]$ forms a parahermitian polynomial matrix [9], for which a generalisation from the EVD to a polynomial EVD (PEVD) exist [5]. In this contribution, we extend the PEVD to a polynomial version of the GEVD (PGEVD) in order to directly address the broadband MWF problem. Wiener filters have previously been formulated using polynomial matrix techniques [6] but initially could not be solved due to an absence of tools. We here completement the polynomial Wiener filter solution in [12] by a PGEVD approach.

Generalised Eigenvalue Decomposition. The GEVD solves the problem of $\mathbf{R}_{1} \mathbf{v}=d \mathbf{R}_{2} \mathbf{v}$, $\mathbf{v} \neq \mathbf{0}$, where $\mathbf{v}$ is an eigenvector of the pencil $\mathbf{R}_{1}-d \mathbf{R}_{2}[2]$. In matrix form,

$$
\mathbf{R}_{1} \mathbf{V}=\mathbf{R}_{2} \mathbf{V D}
$$

the diagonal matrix $\mathbf{D}$ contains the generalised eigenvalues and $\mathbf{V}$ holds the corresponding generalised eigenvectors. When the generalised eigenvectors are applied to $\mathbf{R}_{1}$ and $\mathbf{R}_{2}$ we get

$$
\begin{aligned}
& \mathbf{V}^{\mathrm{H}} \mathbf{R}_{1} \mathbf{V}=\mathbf{D}, \\
& \mathbf{V}^{\mathrm{H}} \mathbf{R}_{2} \mathbf{V}=\mathbf{I},
\end{aligned}
$$

${ }^{\dagger}$ POC: Jamie Corr, Dept. of EEE, University of Strathclyde, Glasgow G1 1XW, Scotland, UK. Tel. 0044(0)141-548-2546 Fax 0044-(0)141-552-2487; e-mail jamie.corr@strath. ac.uk.

This work was supported in part by the Engineering and Physical Sciences Research Council (EPSRC) Grant number EP/K014307/1 and the MOD University Defence Research Collaboration in Signal Processing. 
where $\mathbf{I}$ is the identity. From the alternative form $\mathbf{V}^{\mathrm{H}} \mathbf{R}_{2}^{-1} \mathbf{R}_{1} \mathbf{V}=\mathbf{D}$ to (2) and (3) it is evident that $\mathbf{R}_{2}^{-1} \mathbf{R}_{1}$ is no longer Hermitian and therefore $\mathbf{V}$ not guaranteed to be unitary, even if as in the case of MWF $[3,8]-\mathbf{R}_{1}$ and $\mathbf{R}_{2}$ are Hermitian covariance matrices.

Amongst a number of options to calculate the GEVD or joint diagonalisation of Hermitian matrices $\mathbf{R}_{1}$ and $\mathbf{R}_{2}[2]$ is a Cholesky-based approach. In a first step, a Cholesky decomposition $\mathbf{R}_{2}=\mathbf{L} \mathbf{L}^{\mathrm{H}}$, with $\mathbf{L}$ lower left triangular, is determined. In a second step, an EVD is performed on the intermediate Hermitian matrix $\mathbf{C}=\mathbf{L}^{-1} \mathbf{R}_{1} \mathbf{L}^{-\mathrm{H}}=\mathbf{Y} \mathbf{D Y} \mathbf{Y}^{\mathrm{H}}$, such that $\mathbf{D}$ contains the generalised eigenvalues and the generalised eigenvectors are the columns of $\mathbf{V}=\mathbf{L}^{-\mathrm{H}} \mathbf{Y}$.

Extension of GEVD to Polynomial Matrices. Extending the GEVD in (1) to polynomial parahermitian matrices $\boldsymbol{R}_{i}(z), i=\{1,2\}$, leads to a joint diagonalisation problem akin to (2) and $(3)$

$$
\tilde{\boldsymbol{V}}(z) \boldsymbol{R}_{1}(z) \boldsymbol{V}(z)=\boldsymbol{D}(z), \quad \tilde{\boldsymbol{V}}(z) \boldsymbol{R}_{2}(z) \boldsymbol{V}(z)=\mathbf{I}
$$

where $\boldsymbol{D}(z)$ contains the polynomial generalised eigenvectors. The operator $\{\tilde{\cdot} \cdot\}$ denotes the parahermitian transpose, which includes both a Hermitian transpose and time reversal; this can be seen as the polynomial equivalent of the Hermitian transpose in the scalar case [9]. The factorisations in (4) can be shown to exist if the PEVDs of $\boldsymbol{R}_{i}(z)$ exist [4], and if $\boldsymbol{R}_{2}(z)$ is invertible. Due to non-uniqueness of the PEVD [1], the generalised polynomial eigenvectors in $\boldsymbol{V}(z)$ can at the very least be arbitrarily delayed w.r.t. each other, leading to a variability in their polynomial degree.

For the computation of $\boldsymbol{V}(z)$ and $\boldsymbol{D}(z)$, a two-step Cholesky-style approach can be performed. The first step uses the PEVD $\boldsymbol{R}_{2}(z)=\tilde{\boldsymbol{Q}}_{2}(z) \boldsymbol{D}_{2}(z) \boldsymbol{Q}_{2}(z)$ and the spectral factorisation [10] of $\boldsymbol{D}_{2}(z)$ into its minimum and maximum phase components, $\boldsymbol{D}_{2}^{(+)}(z)$ and $\boldsymbol{D}_{2}^{(-)}(z)=\tilde{\boldsymbol{D}}_{2}^{(+)}(z)$. Therefore the factor $\boldsymbol{L}(z)=\tilde{\boldsymbol{Q}}_{2}(z) \boldsymbol{D}_{2}^{(+)}(z)$ is now not lower left triangular but easily inverted [11], such that $\boldsymbol{L}^{-1}(z)=\left(\boldsymbol{D}_{2}^{(+)}(z)\right)^{-1} \boldsymbol{Q}_{2}(z)$. For the second step, the PEVD $\boldsymbol{C}(z)=\boldsymbol{L}^{-1}(z) \boldsymbol{R}_{1}(z) \tilde{\boldsymbol{L}}^{-1}(z)=\boldsymbol{Q}(z) \boldsymbol{D}(z) \tilde{\boldsymbol{Q}}(z)$ is calculated. This leads to the polynomial generalised eigenvalues on the diagonal of $\boldsymbol{D}(z)$ and the polynomial generalised eigenvectors in $\boldsymbol{V}(z)=\tilde{\boldsymbol{L}}^{-1}(z) \boldsymbol{Q}(z)$, noting a possible order reduction due to ambiguity [1].

Results. As an example of a PGEVD, the source model described in [7] generates two parahermitian matrices $\boldsymbol{R}_{1}(z)$ and $\boldsymbol{R}_{2}(z)$ as shown in Fig. 1. The sequential matrix diagonalisation (SMD) [7] PEVD algorithm is used to decompose $\boldsymbol{R}_{2}(z)$ and to calculate the intermediate matrix $\boldsymbol{C}(z)$. The determined $\boldsymbol{V}(z)$ is then applied to both $\boldsymbol{R}_{1}(z)$ and $\boldsymbol{R}_{2}(z)$, leading to the jointly diagonalised systems in Fig. 2, closely approximating (4).

Conclusions and Full Paper. This summary has introduced the ideas and motivation behind a polynomial matrix generalised eigenvalue decomposition and some initial results from the Cholesky type method based on the polynomial EVD.

The final paper will provide details on the approach and numerical results for joint diagonalisation over an ensemble of differently conditioned parahermitian matrices. These results will be applied to broadband multichannel Wiener filtering, with a comparison to the narrowband GEVD applied in independent frequency bins. We also aim to generalise advantages in the GEVD to the polynomial case if $\boldsymbol{R}_{1}(z)$ has low rank [3], which can be expected to provide simplifications over the polynomial Wiener approach outlined but not solved in [6]. 

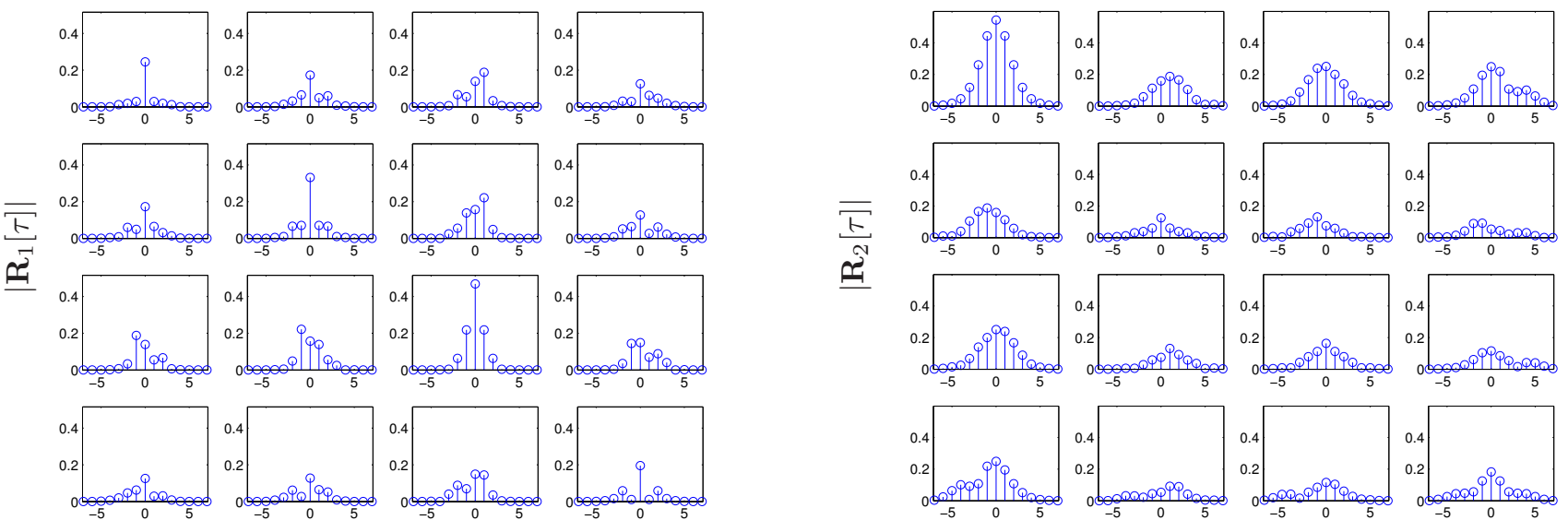

(a)

$\operatorname{lag} \tau$
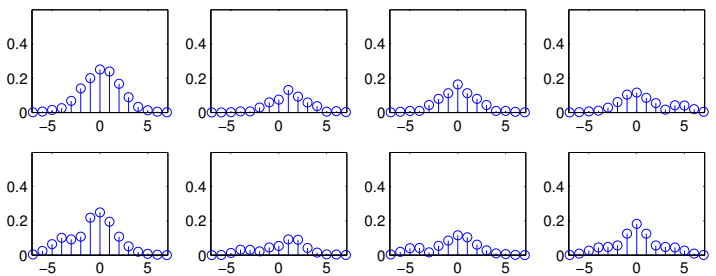

(b)

$\operatorname{lag} \tau$

Fig. 1: Space-time covariance matrix (a) $\mathbf{R}_{1}[\tau] \circ-\bullet \boldsymbol{R}_{1}(z)$ and (b) $\mathbf{R}_{2}[\tau] \circ \cdots \boldsymbol{R}_{2}(z)$, only showing the absolute value of coefficients.

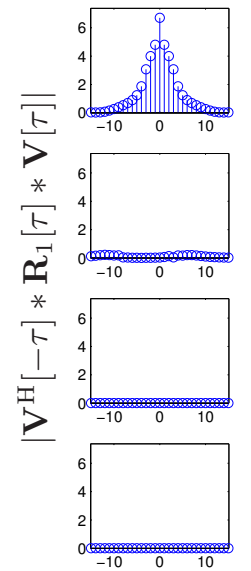

(a)
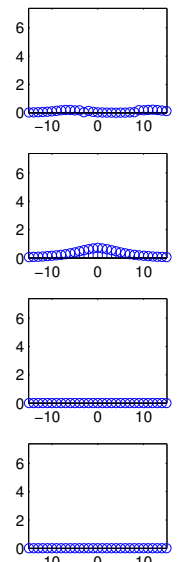

$\operatorname{lag} \tau$
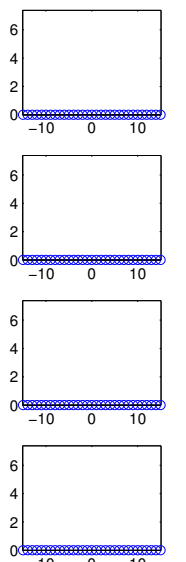

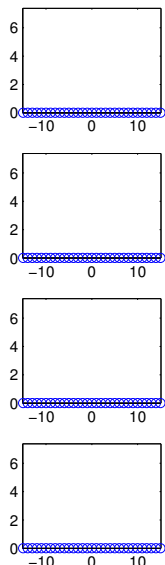

$(\mathrm{b})$

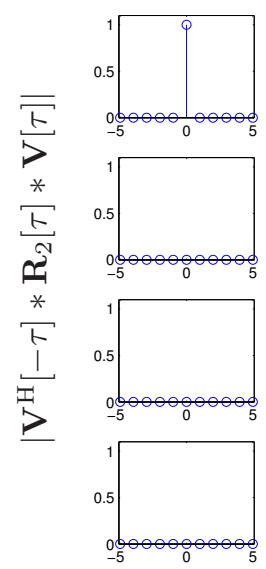

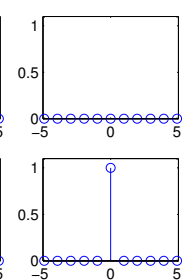
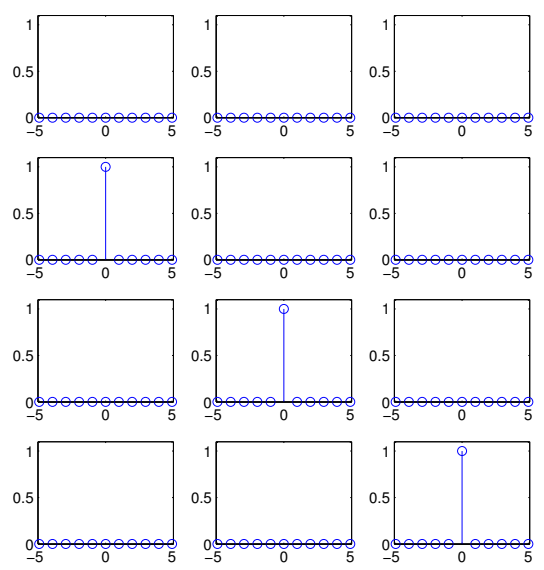

$\operatorname{lag} \tau$

Fig. 2: Results of joint diagonalisation by generalised modal matrix $\boldsymbol{V}(z) \bullet \cdots \mathbf{V}[\tau]$ for (a) $\mathbf{R}_{1}[\tau] \circ-\bullet \boldsymbol{R}_{1}(z)$ and (b) $\mathbf{R}_{2}[\tau] \circ \longrightarrow \boldsymbol{R}_{2}(z)$, only showing the absolute value of coefficients.

\section{References:}

[1] J. Corr, K. Thompson, S. Weiss, I. Proudler, and J. McWhirter. Row-shift corrected truncation of paraunitary matrices for PEVD algorithms. In 23rd European Signal Processing Conference, pages 849-853, Nice, France, August/September 2015.

[2] G. H. Golub and C. F. Van Loan. Matrix Computations. John Hopkins University Press, Baltimore, Maryland, 3rd edition, 1996.

[3] A. Hassani, A. Bertrand, and M. Moonen. Gevd-based low-rank approximation for distributed adaptive node-specific signal estimation in wireless sensor networks. IEEE Transactions on Signal Processing, 64(10):2557-2572, May 2016.

[4] S. Icart and P. Comon. Some properties of Laurent polynomial matrices. In 9th IMA Conference on Mathematics in Signal Processing, Birmingham, UK, December 2012. 
[5] J. G. McWhirter, P. D. Baxter, T. Cooper, S. Redif, and J. Foster. An EVD Algorithm for Para-Hermitian Polynomial Matrices. IEEE Transactions on Signal Processing, 55(5):2158-2169, May 2007.

[6] A. Mertins. MMSE Design of Redundant FIR Precoders for Arbitrary Channel Lengths. IEEE Transactions on Signal Processing, 51(9):2402-2409, September 2003.

[7] S. Redif, S. Weiss, and J. McWhirter. Sequential matrix diagonalization algorithms for polynomial EVD of parahermitian matrices. IEEE Transactions on Signal Processing, 63(1):81-89, January 2015.

[8] R. Serizel, M. Moonen, B. V. Dijk, and J. Wouters. Low-rank approximation based multichannel wiener filter algorithms for noise reduction with application in cochlear implants. IEEE/ACM Transactions on Audio, Speech, and Language Processing, 22(4):785-799, April 2014.

[9] P. P. Vaidyanathan. Multirate Systems and Filter Banks. Prentice Hall, Englewood Cliffs, 1993.

[10] Z. Wang, J. G. McWhirter, and S. Weiss. Multichannel spectral factorization algorithm using polynomial matrix eigenvalue decomposition. In 49th Asilomar Conference on Signals, Systems and Computers, Pacific Grove, CA, November 2015.

[11] S. Weiss, A. Millar, and R. W. Stewart. Inversion of parahermitian matrices. In 18th European Signal Processing Conference, pages 447-451, Aalborg, Denmark, August 2010.

[12] S. Weiss, C. H. Ta, and C. Liu. A wiener filter approach to the design of filter bank based single-carrier precoding and equalisation. In IEEE International Symposium on Power Line Communications and Its Applications, pages 493-498, Pisa, Italy, March 26-28 2007. 